\title{
An ultrasensitive and highly selective determination method for quinones by high-performance liquid chromatography with photochemically initiated luminol chemiluminescence
}

\author{
Sameh Ahmed ${ }^{a, b}$, Naoya Kishikawa ${ }^{a}$, Kaname Ohyama $^{a}$,Toshihide Maki $^{c}$, \\ Hiromasa Kurosaki ${ }^{d}$, Kenichiro Nakashima ${ }^{a}$, Naotaka Kuroda ${ }^{a}$,*
}

${ }^{a}$ Graduate School of Biomedical Sciences, Course of Pharmaceutical Sciences, Nagasaki University, 1-14 Bunkyo-machi, Nagasaki 852-8521, Japan

${ }^{\boldsymbol{b}}$ Department of Pharmaceutical Analytical Chemistry, Faculty of Pharmacy, Assiut University, Assiut 71526, Egypt

c Joint Research Center, Nagasaki University, 1-14 Bunkyo-machi, Nagasaki 852-8521,, Japan

${ }^{d}$ Graduate School of Pharmaceutical Sciences, Kumamoto University, 5-1 Oe-honmachi, Kumamoto 862-0973 , Japan

*Corresponding author;

E-mail address: n-kuro@nagasaki-u.ac.jp

Tel: +81-95-8192894

Fax: +81-95-8192444 


\begin{abstract}
Quinones are a class of compounds of substantial toxicological and pharmacological interest. An ultrasensitive and highly selective chemiluminescence (CL) method was newly developed for the determination of quinones based on the utility of photochemically initiated luminol CL. The method involves ultraviolet (UV) irradiation of quinones to generate reactive oxygen species (ROS) through the unique photosensitization reaction accompanied with the photolytical generation of 3,6-dihydroxyphthalic acid (DHPA) from quinones. The photoproducts were detected by luminol CL reaction. Interestingly, it was noticed that DHPA had enhancement effect for the luminol CL. The generation of the enhancer (DHPA) in association with the oxidant (ROS) in the photochemical reaction greatly increases the sensitivity and selectivity of the proposed luminol CL method. In order to elucidate the type of ROS produced by the photosensitizaion reaction in relation to the proposed CL reaction, we investigated the quenching effect of selective ROS scavengers in the luminol CL. Although several ROS were generated, superoxide anion was the most effective ROS for the generated CL. Moreover, the enhancement mechanism of DHPA for luminol CL was confirmed. The enhancement was found to be through the formation of stabilized semiquinone anion radical that provided long-lived CL. The generation of the semiquinone radical was confirmed by electron spin resonance technique. Furthermore, we developed an HPLC method with on-line photochemical reaction followed by the proposed CL detection for the determination of four quinones. A luminol analogue, L-012, was used for its high sensitivity. The detection limits for quinones obtained with the proposed method $(\mathrm{S} / \mathrm{N}=3)$ were in the range 1.5- $24 \mathrm{fmol}$ that were 10-1000 times more sensitive compared with the previous methods. Finally, the developed HPLC-CL system was successfully applied for the determination of quinones in airborne particulate samples collected at Nagasaki city.
\end{abstract}

Keywords: Quinones; Photochemical reaction; Luminol chemiluminescence; ROS; Enhancer; L-012; Semiquinone radical; ESR. 


\section{Introduction:}

It is well known that quinones play important roles in photosynthesis and photobiology, and apparently the interest in their biological function has stimulated basic chemical research in many scientific fields [1]. They are involved in a huge number of electron transfer processes representing individual steps in a variety of enzymatic systems [2]. Ubiquinone (coenzyme Q10) serves in electron transport chains in mitochondria. Vitamins $\mathrm{K}$, which take part in blood coagulation and bone mineralization processes, have a naphthoquinone structure [3]. In bacteria, pyrroloquinoline quinone is an essential redox cofactor for various dehydrogenase enzymes [4]. Besides, quinones are used clinically for the treatment of a wide variety of malignancies as anthracycline derivatives, which constitute an important class of anticancer drugs [5]. Quinones also contribute to environmental toxicity because of their ability to form covalent bonds with tissue macromolecules and generate reactive oxygen species (ROS), especially superoxide anion and hydroxyl radical, leading to pulmonary oxidative damage [6]. Numerous studies have shown that diesel exhaust particles, which represent a significant contributor to urban airborne particulate matter, exhibit toxicity consistent with quinone-based chemistry [7-10]. Therefore, it is necessary to develop a highly sensitive and selective determination method for monitoring quinones in various fields.

Quantitative analytical methods for quinones have been developed using several techniques including high-performance liquid chromatographic (HPLC) with ultraviolet (UV) [11], fluorescence [12,13], electrochemical [14], chemiluminescence (CL) [15,16], and mass spectrometry (MS) detection [17,18]. Gas chromatography with MS detection was also reported for the determination of quinones [19-25]. Although a variety of analytical techniques exist, many of these techniques lack the enough sensitivity and selectivity which enables the determination of small concentrations of quinones in the complex matrices in which they are typically found [19]. Additionally, the high cost and laborious derivatization procedures limit the use of the others. The popular methods for analysis of quinones cannot meet the continuous demand for the selective and sensitive determination of quinones in complex matrices. Therefore, it is necessary to develop a novel method specially designated for quinones.

Luminol is so far the most frequently used CL reagent. The CL emission of luminol is based on its oxidation in alkaline medium. The relevance of the luminol as analytical CL 
reagent does not rely on emission efficiency but on possibilities that many different species can influence the mechanism and the kinetics of the CL reaction. The analyte can act as enhancer, inhibitor or catalyst whose concentration may influence the intensity or amount of emitted light [26]. The light output of luminol CL reaction can be greatly increased by incorporating different enhancers. Such increase is attributed to the preferential oxidation of the enhancer and the rapid formation of enhancer radicals that, in turn, quickly oxidize luminol to its radical anion. These enhancers, which include substituted phenols, substituted boronic acids, indophenols, and $N$-alkyl phenothiazines, behave as electron transfer mediators [27].

Previously, we reported the determination of quinones by HPLC with peroxyoxalate chemiluminescence (PO-CL) detection combined with on-line photochemical reaction [15] and this method was applied for the determination of vitamin $\mathrm{K}$ homologues [28]. This method was based on the generation of hydrogen peroxide and conversion of non-fluorescent quinones to a fluorescent 3,6-dihydroxyphthalic acid (DHPA) through photodegradation reaction of quinones by UV irradiation, and then detected via PO-CL reaction by mixing with aryloxalate.

In the course of this work, we have exploited the photochemically initiated luminol CL for the determination of quinones. Quinones were subjected to UV irradiation to generate ROS accompanied with the photolytic generation of DHPA from quinones. The photoproducts can initiate luminol CL reaction. It was noticed that DHPA had enhancement effect for luminol CL. The coexistence of oxidant and enhancer could significantly increase the light output and duration kinetics of luminol CL. Hence, this will allow the development of more sensitive and selective analytical methodology for the determination of quinones. To the best of our knowledge, it is the first time to use luminol or its analogues as a single post column CL reagent without addition of oxidant or any catalyst. Till now, luminol is always delivered in association with oxidant and/or catalyst. Furthermore, we elucidated type of the generated ROS in relation to proposed CL reaction, by investigating the quenching effect of selective ROS scavengers in the luminol CL. Also, the enhancement mechanism of DHPA for the luminol CL was confirmed by electron spin resonance (ESR) technique. Then we tried to get further insights on DHPA as a novel enhancer for luminol CL.

Hereby, we developed an HPLC method with on-line photochemical reaction followed by luminol CL detection for the determination of quinones. In this paper, four quinones 
namely; 1,2-naphthoquinone (1,2-NQ), 1,4-naphthoquinone (1,4-NQ), 9,10-phenanthrenequinone (PQ) and 9,10-anthraquinone (AQ) were determined by the proposed $\quad \mathrm{CL}$ method. 8-Amino-5-chloro-7-phenylpyrido[3,4- $d]$ pyridazine $-1,4-(2 H, 3 H)$ dione (L-012), luminol analogue, was tested as a post column CL reagent for its high sensitivity. Subsequently, the developed HPLC system was applied for the determination of quinones in airborne particulate samples collected at Nagasaki city.

\section{Experimental}

\subsection{Materials and reagents}

1,4-Naphthoquinone, 9,10-anthraquinone and luminol were obtained from Nacalai Tesque (Kyoto, Japan). 1,2-Naphthoquinone, 9,10-phenanthrenequinone, isoluminol and $N$-(4-aminobutyl)- $N$-ethyl-isoluminol (ABEI) were from Tokyo Chemical Industry (Tokyo, Japan). L-012 and 3,6-dihydroxyphthalic acid was from Wako Pure Chemical Industries (Osaka, Japan). Potassium superoxide $\left(\mathrm{KO}_{2}\right)$ was from Acros Organics (New Jersey, USA) and sodium hydroxide $(\mathrm{NaOH})$ was from Merck (Darmstadt, Germany). Superoxide dismutase (SOD) from bovine erythrocytes was purchased from Sigma (St. Louis, MO, USA). Stock solutions of quinones $(0.1 \mathrm{mM})$ were prepared in acetonitrile. These solutions were diluted appropriately with mobile phase to prepare the working solutions. Stock solutions of luminol analogues $(0.3 \mathrm{mM})$ were prepared in $100 \mathrm{mM}$ aqueous $\mathrm{NaOH}$ solution and diluted before analysis. A stock solution of $\mathrm{KO}_{2}(10 \mathrm{mM})$ was prepared in acetonitrile and stored together with molecular sieve $4 \AA$ (Nacalai Tesque) to exclude water. Imidazole obtained from Tokyo Chemical Industry was recrystallized from acetonitrile before use. Distilled water was obtained using Simpli Lab-UV (Millipore, Bedford, MA, USA) water device. Other chemicals were of extra pure grade.

\subsection{Confirmation of ROS generated from photochemical reaction}

To confirm type of the generated ROS in relation to the proposed CL reaction, we investigated the quenching effect of selective ROS scavengers for the CL produced by the photoproducts with L-012. A $10 \mu \mathrm{M}$ of $\mathrm{AQ}$, dissolved in a mixture of imidazole-trifluoroacetic acid (TFA) (100 mM, pH 9.0) and acetonitrile (1:1), was UV 
irradiated in a photoreactor composed of a PTFE tubing $(5.0 \mathrm{~m} \times 0.25 \mathrm{~mm}$ i.d.,) coiled around the quartz well of Philips TUV-SE low-pressure mercury lamp (16 W, $254 \mathrm{~nm}$, Philips, Poland). In a small test tube, $100 \mu \mathrm{L}$ of photoproducts was mixed with the ROS scavengers. SOD (1 and $10 \mathrm{U} / \mathrm{mL})$, mannitol $(10$ and $100 \mu \mathrm{M})$ and sodium azide (10 and $100 \mu \mathrm{M})$ were used as selective scavengers for superoxide anion radical $\left(\mathrm{O}_{2} \cdot-\right.$, hydroxyl radical $\left({ }^{\circ} \mathrm{OH}\right)$ and singlet oxygen $\left({ }^{1} \mathrm{O}_{2}\right)$, respectively. The test tube was immediately placed in Sirius luminometer (Berthold, Germany) and then $100 \mu \mathrm{L}$ of $3 \mu \mathrm{M}$ L-012 in $100 \mathrm{mM}$ $\mathrm{NaOHaq}$ was injected automatically, and the produced CL was measured for $600 \mathrm{~s}$. All mixing and reagent addition steps were carried out rapidly. The total CL intensity was defined as the area under the CL delay curve. Data was expressed as the mean of triplicate measurements.

\subsection{Confirmation of the role of DHPA in luminol CL}

The working solution of standard DHPA was freshly prepared in a mixture of imidazole-TFA (pH 9.0) and acetonitrile (1:1). In order to confirm the role of DHPA in CL, $100 \mu \mathrm{L}$ of freshly prepared DHPA standard solution $(100 \mu \mathrm{M})$ was added in test tube placed in Sirius luminometer and then $100 \mu \mathrm{L}$ of $3 \mu \mathrm{M}$ L-012 in $100 \mathrm{mM} \mathrm{NaOHaq}$ was injected automatically. The produced CL was measured for $600 \mathrm{~s}$. The CL reaction kinetics was carried out under aerobic conditions. The effect of different concentrations of DHPA in the presence of $\mathrm{O}_{2}{ }^{\bullet-}$ was examined. $\mathrm{O}_{2}{ }^{\bullet}-$ was generated by spontaneous reaction between $\mathrm{KO}_{2}$ and water. To a test tube containing $100 \mu \mathrm{L}$ of freshly prepared DHPA standard solution ( 0 , 20, 50 and $100 \mu \mathrm{M}), 100 \mu \mathrm{L}$ of $\mathrm{KO}_{2}$ solution $(21.3 \mu \mathrm{g} / \mathrm{mL})$ in anhydrous acetonitrile was added. The test tube was placed in Sirius luminometer and then $100 \mu \mathrm{L}$ of $3 \mu \mathrm{M} \mathrm{L}-012$ in $100 \mathrm{mM} \mathrm{NaOHaq}$ was injected automatically. The produced CL was measured for $600 \mathrm{~s}$ then the total CL intensity was calculated $(n=3)$. All mixing and reagent addition steps were carried out rapidly.

\subsection{ESR measurement of semiquinone radical of DHPA}

The ESR spectra of all samples were measured using JES-TE 200 ESR spectrometer (JEOL, Tokyo, Japan). Typical ESR parameters were as follows: power, $1.26 \mathrm{~mW}$; frequency, 9.44 GHz; field, $335.2 \mathrm{mT}$; mod width, $0.25 \mathrm{mT}$; $\bmod$ frequency $100 \mathrm{kHz}$, sweep time, $4 \mathrm{~min}$; 
time constant, $0.3 \mathrm{~s}$. ESR spectra were recorded at room temperature. The g-values of ESR spectra were calculated using $\mathrm{Mn}^{2+}$ as a reference $\left(g_{1} 1.981\right.$ and $\left.g_{2} 2.034\right)$ by interpolation between the $\mathrm{Mn}^{2+}$ signals. The working solution of standard DHPA $(100 \mu \mathrm{M})$ was freshly prepared in a mixture of imidazole-TFA ( $\mathrm{pH} 9.0)$ and acetonitrile $(1: 1)$. The effect of $\mathrm{O}_{2}{ }^{\bullet}$ - on ESR spectra of DHPA was studied using $35.5 \mu \mathrm{g} / \mathrm{mL}$ of $\mathrm{KO}_{2}$ in anhydrous acetonitrile. Moreover, semiquinone anion radical was detected in the photoproducts of different quinones. Quinone solutions $(20 \mu \mathrm{M})$, dissolved in a mixture of imidazole-TFA ( $\mathrm{pH} 9.0)$ and acetonitrile (1:1), were UV irradiated in the photoreactor (described in section 2.2.). The photoproducts were collected and measured by ESR spectrometer.

The theoretical calculations of spin density of semiquinone anion radical of DHPA were performed by WinMOPAC (version 3.0) program using PM3 as a semiempirical hamiltonian method.

\section{5. HPLC-CL system}

The chromatographic analysis was performed at ambient temperature $\left(25^{\circ} \mathrm{C}\right)$ on a Discovery HS C18 (250 mm $\times 4.6 \mathrm{~mm}$ i.d. $5 \mu \mathrm{m}$, Supelco, Sigma-Aldrich, USA); the HPLC system consisted of two LC 10AS liquid chromatographic pumps (Shimadzu, Kyoto, Japan), a Rheodyne 7125 injector (Cotati, CA, USA) with a 20- $\mu$ l sample loop, an online photoreactor (described in section 2.2) placed after the column, temperature of the photoreactor was maintained at $25^{\circ} \mathrm{C}$ by an oven (Jasco, Tokyo), a CLD-10A CL detector (Shimadzu) and SIC chromatorecorder (Tokyo, Japan). A mixture of imidazole-TFA (100 $\mathrm{mM}, \mathrm{pH}$ 9.0) and acetonitrile (1:1) was used as a mobile phase and 3- $\mu \mathrm{M} \mathrm{L-012} \mathrm{in} 0.1 \mathrm{M}$ $\mathrm{NaOHaq}$ was used as a single post column CL reagent. The flow-rates of the mobile phase and the CL reagent were set at 0.75 and $0.8 \mathrm{ml} / \mathrm{min}$, respectively

\subsection{Airborne particulates sample}

Sampling of airborne particulates was carried out at the main avenue of Nagasaki City. Airborne particulates were collected on Q-R100 silica-fiber filters (Advantec Toyo, Tokyo, Japan) at a flow rate of $1200 \mathrm{l} / \mathrm{min}$ for $24 \mathrm{~h}$ by a Model No. 120 FT type high-volume air sampler (Kimoto Electric, Osaka, Japan). The filters containing airborne particulates were stored at $-30^{\circ} \mathrm{C}$ in the dark until analysis. The filter $(1 \mathrm{~cm} \mathrm{x1} \mathrm{cm)} \mathrm{was} \mathrm{extracted}$ 
ultrasonically with $4 \mathrm{ml}$ of a mixture of dichloromethane and methanol (1:1) for $20 \mathrm{~min}$. After taking the organic layer, the extraction was repeated again on the same filter. These organic layers were combined and evaporated to dryness, and the resultant residue was dissolved in $100 \mu \mathrm{l}$ of methanol. The reconstitute solution was injected directly to HPLC-CL system. The recovery experiments were carried out by spiking $10 \mu \mathrm{L}$ of $500 \mathrm{nM}$ of standard quinones mixture to airborne particulate filter. After drying, the filter was treated as described before and the results were compared with that without spiking. The concentration differences were compared to spiked concentrations of quinones.

\section{Results and discussion}

\subsection{Confirmation of ROS generated from photochemical reaction}

Quinones are known for their unique photosensitizing properties [29]. The photosensitizer (S) absorbs light to become a singlet-excited state $\left({ }^{1} \mathrm{~S}\right)$, which is rapidly converted into its triplet-excited state $\left({ }^{3} \mathrm{~S}\right)$ by intersystem crossing. The photoexcited quinones can produce ROS via two competing reactions. In type I reactions, ${ }^{3} \mathrm{~S}$ participates in electron transfer or hydrogen abstraction reactions with nearby molecules, followed by fast addition of $\mathrm{O}_{2}$ to produce $\mathrm{O}_{2}{ }^{\bullet-},{ }^{\bullet} \mathrm{OH}$ or $\mathrm{H}_{2} \mathrm{O}_{2}$. Whereas in type II reaction, ${ }^{3} \mathrm{~S}$ may transfer energy directly to $\mathrm{O}_{2}$ to form electronically excited singlet state of oxygen ${ }^{1} \mathrm{O}_{2}$ [29-31].

Herein, we tried to get further insights on the photogenerated ROS and their role in the proposed CL reaction. The photogenerated $\mathrm{O}_{2}{ }^{\bullet},{ }^{\circ} \mathrm{OH}$ and ${ }^{1} \mathrm{O}_{2}$ were confirmed by using selective ROS scavengers. We investigated the quenching effect of selective ROS scavengers on $\mathrm{CL}$ generated from the reaction between anthraquinone photoproducts and L-012 in alkaline medium. SOD, mannitol and sodium azide were used as selective scavengers for $\mathrm{O}_{2}{ }^{\bullet},{ }^{\bullet} \mathrm{OH}$ and ${ }^{1} \mathrm{O}_{2}$, respectively (Table 1). It was clear that extreme quenching for the produced CL was noticed in the presence of SOD whereas smaller quenching effect was found for mannitol and sodium azide. A similar quenching effects were obtained for all the studied quinones. Therefore, $\mathrm{O}_{2}{ }^{-}$was the main species that involved in the generated CL reaction. The other photogenerated ROS species are either in small amounts or less effective for the generated CL. The smaller amount of the photogenerated ${ }^{1} \mathrm{O}_{2}$ may be attributed to the bleaching effect of imidazole on ${ }^{1} \mathrm{O}_{2}$ [31]. Recently, we have reported the photolytic generation of DHPA from UV irradiation of 
quinones [15]. It was found that DHPA was generated from four different forms of quinones only in the presence of imidazole. It was assumed that quinones were initially decomposed to phthalic acid that may interact with the photogenerated ${ }^{\circ} \mathrm{OH}$ to form DHPA and this reaction was catalyzed by imidazole. Hence, there was a possibility for the consumption of •OH for the photogeneration of DHPA. However, the mechanism is still not well understood therefore, several studies are required to clarify this mechanism. Moreover, we previously investigated the generation of hydrogen peroxide after UV irradiation of quinone and its role in PO-CL reaction [15]. However, luminol CL in the presence of hydrogen peroxide can be produced through the action of a chemical catalyst like ferricyanide or a biocatalyst such as peroxidase to convert hydrogen peroxide into more active species [32]. Thereby, ROS generated by UV irradiation of the quinones and its role in the CL reaction became clear.

\subsection{Confirmation of the role of DHPA in luminol CL}

We have studied the effect of DHPA standard solution on the kinetics and profile of the CL reaction (Fig. 1A). A strong CL was emitted after injection of L-012 to standard DHPA in alkaline medium and continued for more than 10 min indicating a long-lived CL. The produced CL was significantly quenched by addition of SOD indicating the formation of $\mathrm{O}_{2}{ }^{\bullet}$. DHPA is capable of enhancing the intensity of CL based on the fact that hydroquinone is auto-oxidized under aerobic conditions in alkaline medium to generate semiquinone anion radical and $\mathrm{O}_{2}{ }^{-}$[33-35]. However, in case of UV irradiated quinones both DHPA and $\mathrm{O}_{2}{ }^{-}$might be generated. Hence there are two different sources for $\mathrm{O}_{2}{ }^{-}$; from the photosensitization reaction of quinones and also from auto-oxidation reaction of the generated DHPA. In addition, the CL profile of DHPA with L-012 was found to be similar to those obtained by UV irradiated quinones with L-012.

The generated semiquinone radical anion might act as an enhancer for luminol CL via its ability to react with luminol monoanion $\left(\mathrm{LH}^{-}\right)$to produce the key intermediate luminol radical anion $\left(\mathrm{L}^{\bullet}\right)$. The long-lived $\mathrm{CL}$ phenomenon presumed the formation of a persistent enhancer radical. The generated semiquinone anion radical was effective in the primary oxidation step whereas $\mathrm{O}_{2}{ }^{\bullet}-$ works well in the secondary oxidation step of $\mathrm{L}^{\bullet}-$ to luminol endoperoxide. The produced endoperoxide decomposes into electronically excited aminophthalate that decays to the ground state to produce luminescence [36].

Moreover, the photochemical reaction involved the generation of $\mathrm{O}_{2}^{\bullet-}$ as the main ROS 
species. $\mathrm{O}_{2}{ }^{-}-$was reported to be ineffective in the primary oxidation step but play a significant role in the secondary oxidation step of luminol radical [37]. Therefore, the effect of $\mathrm{O}_{2}{ }^{-}-$on the time profiles of CL emission for different concentrations of DHPA standard solution with L-012 was also investigated (Fig. 1B). As shown, the presence of photogenerated ROS such as $\mathrm{O}_{2}{ }^{\bullet}$ - increased significantly the generated CL. It was noticed that the CL intensity increased by increasing concentration of DHPA while the time required to reach the maximum was not changed. This can be explained by the rapid reaction of the generated semiquinone anion radical and $\mathrm{O}_{2}{ }^{\bullet-}$ with luminol. The total $\mathrm{CL}$ intensity was increased as a function of DHPA concentration. The long-lived CL indicated the formation of a stabilized semiquinone radical for DHPA.

The formation of semiquinone anion radical from standard DHPA at alkaline $\mathrm{pH}$ was evidenced by ESR technique (Fig. 2A). The ESR studies were carried out at pH of 9.0 using imidazole buffer. These conditions were suitable for the generation of semiquinone radical. However, higher $\mathrm{pH}$ often leads to side reactions [35]. The ESR spectrum of DHPA semiquinone radical anion shows a singlet with no hyperfine coupling constant. This spectrum shows a quite distinctive semiquinone radical structure with $g=2.0047 \pm 0.0005$.

We further investigated the photogeneration of semiquinone radical of DHPA after photolysis of anthraquinone (Fig. 2B). A similar spectrum was obtained for all the studied quinones with $g=2.0042 \pm 0.0006$. Moreover, the spin density of semiquinone anion radical of DHPA was calculated using PM3 as a semiempirical hamiltonian method (Fig. $2 \mathrm{C})$. The theoretical calculations reveal the formation of two symmetrical intramolecular hydrogen bondings (IHB) (1.726 $\AA$ ). The presence of IHB induces a higher degree of localization of spin density at postion 3 and 6 . Furthermore, the formation of IHB leads to stabilization of the generated semiquinone radical. We also examined the effect of $\mathrm{O}_{2}{ }^{\bullet-}$ on ESR spectrum of DHPA using $35.5 \mu \mathrm{g} / \mathrm{mL}$ of $\mathrm{KO}_{2}$ in anhydrous acetonitrile. As expected, $\mathrm{O}_{2}{ }^{\bullet-}$ accelerated the formation of semiquinone anion radical and the ESR signal intensity was increased.

\subsection{Suggested photochemically initiated luminol CL mechanism}

The suggested mechanism for the photochemically initiated luminol CL of quinones is shown in Fig 3. Quinones were subjected to photochemical reaction to generate DHPA and ROS (mainly $\mathrm{O}_{2}^{\bullet}$ ). Then the generated photoproducts were mixed with luminol. In 
alkaline medium, $\mathrm{LH}^{-}$is formed by ketoenol tautomerism and subsequently a simple ionization reaction. It was reported that mechanism of luminol CL reaction involved two successive oxidation steps, which are primary and secondary oxidation reactions [37]. The primary oxidation of luminol can be achieved via many oxidants and free radicals to yield luminol anion radical $\left(\mathrm{L}^{\bullet} \sqsupset\right.$ ). DHPA may be transformed via auto-oxidation process into stabilized semiquinone anion radical and $\mathrm{O}_{2}{ }^{-}$. It is assumed that semiquinone anion radical might act as an electron mediator by reaction with $\mathrm{LH}^{-}$to produce $\mathrm{L}^{\bullet}-$ and thereby initiate luminol CL reaction whereas $\mathrm{O}_{2}{ }^{\bullet}-$ works well in the secondary oxidation step [37]. The results strongly support the role of semiquinone radical anion in the primary oxidation step. The secondary oxidation step involves the reaction between $\mathrm{L}^{\bullet}$ and the generated $\mathrm{O}_{2}{ }^{-}$to produce endoperoxide. However, $\mathrm{O}_{2}{ }^{-}$is from two different origins; one of them is from the photosensitization reaction of quinones and the other from auto-oxidation reaction of the generated DHPA. The transient luminol endoperoxide decomposes into electronically excited aminophthalate. Luminescence occurs at $425 \mathrm{~nm}$ when the aminophthalate decays to the ground state [32]. Accordingly, the results support the entity of coexisted oxidant and enhancer in the proposed CL method. Therefore, it is conceivable that the coexistence of oxidant and enhancer allowed the proposed CL method to be highly sensitive and selective without the addition of any oxidant and/or catalyst.

\subsection{Optimization of HPLC-CL method}

\subsubsection{Optimization of photochemical reaction conditions}

It is thought that the photochemical reaction plays an important role in the CL reaction of quinones through the generation of highly reactive photoproducts. The reaction photoproducts are highly dependent on residence time in the photoreactor, temperature of photochemical reactor, $\mathrm{pH}$ and composition of the mobile phase [38]. Therefore, the photochemical reaction parameters were optimized in order to get the maximum sensitivity for the proposed CL method. At first, the effect of PTFE coil length on CL intensity was investigated. PTFE coil ranging from 2.0 to $8.0 \mathrm{~m}$ corresponding to $8-32$ seconds of residence time in the photoreactor was examined. It was found that CL increased as the length of the reaction coil increased due to the increase in exposure time then it decreased. This may be attributed to the simultaneous generation and decomposition of ROS and 
DHPA due to long residence time. Moreover, the detection sensitivity ( $\mathrm{S} / \mathrm{N}$ ratio) was measured against coil length for all samples. It was found that $\mathrm{S} / \mathrm{N}$ ratio increase by increasing coil length till maximum then decrease. The optimum coil length was found to be $5 \mathrm{~m}$ corresponding to 20 seconds of residence time.

Since the temperature of photochemical reaction strongly affects the generation efficiency and stability of photogenerated oxygen species and DHPA, the effect of photoreactor temperature from $20-50^{\circ} \mathrm{C}$ on $\mathrm{CL}$ intensity and $\mathrm{S} / \mathrm{N}$ ratio was examined. There was a significant increase in CL intensity with temperature increase. However, there was no significant change in $\mathrm{S} / \mathrm{N}$ ratio with the rise in temperature because the background noise level rose at the same time so, $25{ }^{\circ} \mathrm{C}$ was selected.

The mobile phase compositions play an important role in the photochemical reaction kinetics and reaction pathways [38]. The photochemical degradation of quinones to DHPA was catalyzed by imidazole. Therefore, the concentration and $\mathrm{pH}$ of imidazole buffer were optimized. A mixture of imidazole-TFA buffer/acetonitrile (1:1) was sufficient for complete separation of all the studied quinones. The effect of imidazole concentration on the CL intensity and $\mathrm{S} / \mathrm{N}$ ratio was examined in the range $25-150 \mathrm{mM}$. It was found that both $\mathrm{CL}$ intensity and $\mathrm{S} / \mathrm{N}$ ratio increased by increasing concentration till $100 \mathrm{mM}$ then decreased. Therefore, $100 \mathrm{mM}$ of imidazole-TFA solution was chosen. Moreover, $\mathrm{pH}$ of imidazole buffer solution significantly affects the photochemical reaction and hence the photogenerated products. A buffer solution with $\mathrm{pH}$ 6.0-10.0 was investigated for its effect on $\mathrm{CL}$ intensity and $\mathrm{S} / \mathrm{N}$ ratio. It was found that both $\mathrm{CL}$ intensity and $\mathrm{S} / \mathrm{N}$ ratio increased as a function of $\mathrm{pH}$. However, column durability limits the use of higher $\mathrm{pH}$ therefore, $\mathrm{pH}$ of 9.0 was selected for subsequent work.

\subsubsection{Optimization of CL conditions}

The factors influencing the CL intensity such as type of luminol analogues, $\mathrm{pH}$ of the solutions, concentration of luminol analogue and $\mathrm{NaOH}$, and flow rate of $\mathrm{CL}$ reagent were studied in order to obtain maximal detection sensitivity.

So far, several luminol analogues were reported [39]. Among them, luminol, isoluminol, ABEI and L-012 revealed highly efficient and strong CL properties. In this experiment, we studied the effect of these analogues on $\mathrm{CL}$ intensity and $\mathrm{S} / \mathrm{N}$ ratio (Fig. 4). Although isoluminol and $\mathrm{ABEI}$ gave low background noise, $\mathrm{S} / \mathrm{N}$ ratio was far lower compared with 
luminol and L-012. Among them, L-012 gave the best CL intensity and S/N ratio. Moreover, L-012 revealed high sensitivity and selectivity for the detection of $\mathrm{O}_{2}{ }^{\bullet-}[40]$. Therefore, L-012 was the most convenient luminol analogue for the determination of quinones by the proposed method.

The influence of the L-012 concentration on CL intensity and S/N ratio was also examined (Fig. 5). It was found that CL intensity increased linearly as the concentration of L-012 increased however, the background noise was also increased. The best $\mathrm{S} / \mathrm{N}$ ratio was found at $3 \mu \mathrm{M}$ of L-012. This was probably due to the poor catalytic effect of interferences at low concentration of L-012. The elimination of interferences will reduce background noise and enable the method high selectivity.

It is well known that luminol CL reaction takes place in alkaline medium and the degree of alkalinity has great influence on CL intensity. The activity of a chemical enhancer, which is the most attractive mode for increasing the sensitivity, is strongly dependant upon $\mathrm{pH}$ of the $\mathrm{CL}$ reaction system $[41,42]$. Therefore, the effect of $\mathrm{pH}$ on CL reaction of L-012 was investigated. L-012 was dissolved in $\mathrm{NaOH}$ solution of different concentrations ranging from 1-250 $\mathrm{mM}$ corresponding to $\mathrm{pH}$ range 11.0-13.4. CL intensity and $\mathrm{S} / \mathrm{N}$ ratio reach maximum value when the concentration of $\mathrm{NaOH}$ was $100 \mathrm{mM}$ then it became constant. Therefore, the concentration of $100 \mathrm{mM} \mathrm{NaOH}$ was selected. Because the light output of luminol type CL reaction is time dependent, the effect of flow rate of L-012 solution as a single post column reagent was finally examined. It was found that CL intensity increased by the increase in flow rate and reached maximum at $0.8 \mathrm{ml} / \mathrm{min}$ whereas the noise remain constant then CL intensity decreased. This was probably due to the decrease in the reaction time between L-012 and photogenerated reactive species. Thus, flow rate of $0.8 \mathrm{ml} / \mathrm{min}$ was the optimal.

\subsection{Validation of the proposed method}

Under the optimum experimental conditions, linear relationship was observed by plotting relative chemiluminescence intensity (RCI) against quinone concentration (nM). Calibration curve, retention time, calibration range, correlation coefficient and detection limit were recorded for each quinone (Table 2). The calibration graphs were linear in the range 0.2-1000 $\mathrm{nM}$ with good correlation coefficients $(>0.998)$. Also, the detection limits $(\mathrm{S} / \mathrm{N}=3)$ for quinones obtained with the proposed method were in the range $1.5-24 \mathrm{fmol}$. 
The proposed method was found to be more sensitive than most of the reported methods. It was 1000 times more sensitive compared with GC-MS method [19], 10-600 times compared with LC-MS [17-18] and 12-120 times compared with CL methods [15-16]. The order of CL strength and detection sensitivity for all the studied quinones after the photochemical reaction were as follows; $\mathrm{AQ}>\mathrm{PQ}>1,4-\mathrm{NQ}>1,2-\mathrm{NQ}$. Fig. 6 shows a typical chromatogram of a standard mixture of quinones $(50 \mathrm{nM})$ using the proposed HPLC-CL method and under the optimum conditions. The studied quinones were separated efficiently and all peaks were eluted within 30 minutes.

The intra and inter-day precision of the proposed method were examined using standard quinones solutions at three different concentration levels; low (10 nM), middle (100 nM) and high $(500 \mathrm{nM})$ concentrations. It was found that the relative standard deviations intra-day $(n=5)$ and inter-day $(n=3)$ were $0.9-4.6 \%$ and $3.6-6.1 \%$, respectively so excellent reproducibility was obtained.

\subsection{Determination of quinones in airborne particulates samples}

In order to test the usefulness of the proposed HPLC-CL method for the monitoring of quinones in real samples, the method was applied for the determination of quinones in airborne particulates samples collected at Nagasaki city. To choose the extraction solvent, the extraction by ultrasonication was examined with methanol, acetonitrile, dichloromethane, chloroform, ether, hexane and a mixture of dichloromethane and methanol (1:1). Although high extractabilities were obtained for some quinones by methanol and dichloromethane, the best extractabilities for all the studied quinones were obtained with a mixture of dichloromethane and methanol (1:1). Additionally, the effect of sonication time on the extractabilities was studied. The maximum extractabilities were attained by sonication for more than $20 \mathrm{~min}$. The number of repeated extractions was also investigated. Maximum and constant extractabilities were obtained with two or more extraction folds. Therefore, the extraction was performed twice on the same filter. Under the optimal extraction conditions, the average extraction recoveries for all the studied quinones were $81.4-92.8 \%$.

Fig. 7 represents chromatograms obtained with the extract from airborne particulates (A) and that of airborne particulates samples with $50 \mathrm{nM}$ of a mixture of standard quinones (B). It is clear from the chromatograms that the studied quinone can be detected without any 
interference. The chromatograms indicate the high selectivity and sensitivity of the proposed CL method. The average concentration $(n=3)$ of quinones found in airborne particulates collected at Nagasaki city were $0.11,0.24,0.05,0.06 \mathrm{ng} / \mathrm{m}^{3}$ for AQ, PQ, 1,4-NQ and 1,2-NQ, respectively. These results are well in accordance with the published data $[12,19]$.

\section{Conclusion}

Herein, we reported an ultrasensitive and highly selective HPLC method with a photochemically initiated luminol CL detection for simultaneous determination of quinones. The proposed method involved the generation of ROS based on the unique photosensitization reaction of quinones while quinones were simultaneously converted into DHPA through photolytical reaction. The photogeneration of the enhancer (DHPA) in association with the oxidant (ROS) in that photochemical reaction greatly increases the light output and duration kinetics of luminol CL. This phenomenon allowed the development of a highly sensitive and selective analytical methodology for the determination of quinones. The proposed HPLC-CL method was 10-1000 times more sensitive compared with the reported methods [15-19]. Because the luminol analogue, L-012, was used as a single post column reagent without the addition of oxidant or any catalyst, the decrease in the background noise could be expected. In addition, the proposed method does not require a time-consuming labeling procedure and has satisfactory reproducibility and sensitivity. Besides, DHPA was found to be a novel enhancer for luminol CL that could be used for several applications. The proposed HPLC-CL method was successfully applied to the determination of quinones in airborne particulates samples with a simple extraction procedure. Therefore, the novel luminol CL method should be useful for monitoring of quinones in environmental and biological samples.

\section{References}

[1] J. Barber, Biochim. Biophys. Acta,1365 (1998) 269.

[2] I. Gutierrez, S. G. Bertolotti, M. A. Biasutti, A. T. Soltermann, N. A. Garcia, Can. J. Chem. 75 (1997) 423.

[3] M. J., Shearer, Lancet, 345 (1995) 229. 
[4] J. A. Duine, J. Biosci. Bioeng., 88(1999) 231.

[5] G.N. Hortobagyi, Drugs, 54 (Suppl. 4) (1997) 1.

[6] T. R. Henry, K. B. Wallace, Arch. Toxicol. 70 (1996) 482.

[7] Y. Bai, A.K. Suzuki, M. Sagai, Free Radic. Biol. Med. 30 (2001)555.

[8] N. Kishikawa, M. Nakao, Y. Ohba, K. Nakashima, N. Kuroda, Chemosphere, 64 (2006) 834.

[9] A. Mallakin, D. G. Dixon, B. M. Greenberg, Chemosphere, 40 (2000) 1435.

[10] A. Mallakin, B. J. McConkey, G. Miao, B. McKibben, V. Snieckus, D. G. Dixon, B. M. Greenberg, Ecotoxicol. Environ. Saf., 43 (1999) 204.

[11] A. Valavanidis, K. Fiotakis, T. Vlahogianni, V. Papadimitriou, V. Pantikaki, Environ. Chem, 3 (2006) 118.

[12] N. Kishikawa, M. Wada, Y. Ohba, K. Nakashima, N. Kuroda, J. Chromatogr. A, 1057 (2004) 83.

[13] J. R. Poulsen and J. W. Birks, Anal. Chem., 61 (1989) 2267.

[14] M. T. Galceran, E. Moyano, Talanta, 40 (1993) 615.

[15] S. Ahmed, S. Fujii, N. Kishikawa, Y. Ohba, K. Nakashima, N. Kuroda, J. Chromatogr. A, 1133 (2006) 76.

[16] J. R. Poulsen, J. W. Birks, Anal. Chem., 62 (1990) 1242.

[17] C. A. Jakober, M. J. Charles, M. J. Kleeman, P. G. Green, Anal. Chem., 78 (2006) 5086.

[18] J. Lintelmann, K. Fischer, E. Karg, A. Schroppel, Anal. Bioanal. Chem., 381 (2005) 508.

[19] A. K. Cho, E. D. Stefano, Y. You, C. E. Rodriguez, D. A. Shmitz, Y. Kumagai, A. H. Miguel, A. E.-Fernandez, T. Kobayashi, E. Avol and J. R. Froines, Aerosol Sci. Technol., 38 (2004) 68.

[20] M. Y. Chung, R. A. Lazaro, D. Lim, J. Jackson, J. Lyon, D. Rendulic, A. S. Hasson, , Environ. Sci. Technol., 40 (2006) 4880.

[21] M. Maria del Rosario Sienra, Atmos. Environ., 40 (2006) 2374.

[22] C. A. Jakober, S. G. Riddle, M. A. Robert, H. Destaillats, M. J. Charles, P. G. Green, M. J. Kleeman, Environ. Sci. Technol., 41 (2007) 4548.

[23] S. Nicol, J. Dugay, M. C. Hennion, Chromatographia, 53 (2001) S-464.

[24] D. R. Choudhury, Environ. Sci. Technol., 16 (1982) 102.

[25] A. E.-Fernandez, A. H. Miguel, E. D. Stefano, D. A. Shmitz, A. K. Cho, S. Thurairatnam, E. Avol and J. R. Froines, Aerosol Sci. Technol., 42 (2008) 854. 
[26] K. Mervartova, M. Polasek, J. Martinez Calatayud, J. Pharm. Biomed. Anal., 45 (2007) 367.

[27] E. Marzocchi, S. Grilli, L. Ciana, Luca Prodi, M. Mirasoli, A. Roda, Anal. Biochem., 377 (2008) 189.

[28] S. Ahmed, N. Kishikawa, K. Nakashima, N. Kuroda, Anal. Chim. Acta, 591 (2007) 148.

[29] S. C. Montoya, L. R. Comini, M. Sarmiento, C. Becerra, I. Albesa, G. A. Arguello, J. Cabrera, J. Photochem. Photobiol. B: Biol., 78 (2005) 77.

[30] M. Rajendran, R. Gradhidasan, R. Murugesan, J. Photochem. Photobiol. A: Chem., 168 (2004) 67.

[31] K. Mothilal, J. Inbaraj, C. Chignell, R. Gradhidasan, R. Murugesan, J. Photochem. Photobiol. A: Chem., 163 (2004) 141.

[32] C. Lu, G. Song and J.M. Lin, Trend Anal. Chem. 25 (2006), 985.

[33] P. Ever, Chem. Biol. Interact., 80 (1991)159.

[34] K. Ollinger, G. D. Buffinton, L. Ernster, E. Cadenas, Chem. Biol. Interact., 73(1990) 53.

[35] J. A. Pedersen, Spectrochim Acta, Part A, 58 (2002) 1257.

[36] J. Lind, G. Merenyi, T. E. Eriksen, J. Am. Chem. Soc., 105 (1983) 7655.

[37] L. R. Andrew, T. W. David, Anal. Chem., 73 (2001) 5909.

[38] J. W. Birks, R. W. Frei, Trend Anal. Chem.. 1 (1982), 361.

[39] C. Dodeigne, L. Thunus, R. Lejeune, Talanta, 51 (2000) 415.

[40] I. Imada, E. F. Sato, M. Miyamoto, Y. Ichimori, Y. Minamiyama, R. Konaka, M. Inoue, Anal. Biochem., 271 (1999) 53.

[41] H. Xu, C. F. Duan, C. Z. Lai, M. Lian, Z. F. Zhang, L. J. Liu, H. Cui, Luminesence, 21 (2006) 195.

[42] H. Cui, M. J. Shi, R. Meng, J. Zhou, C. Z. Lai, X. Q. Lin., Photochem. Photobiol., 79 (2004) 233. 


\section{Figures Caption}

Fig. 1. Time profiles of CL emission obtained by (A) standard DHPA in alkaline solution (B) different concentrations of standard DHPA with $\mathrm{O}_{2}{ }^{-}-(21.3 \mu \mathrm{g} / \mathrm{mL})$. The used concentration of L-012 was $3 \mu \mathrm{M}$.

Fig. 2. ESR spectrum of semiquinone anion radical formed by (A) DHPA $(100 \mu \mathrm{M})$ in alkaline medium (B) UV irradiation of anthraquinone solution $(20 \mu \mathrm{M})$. (C) spin distribution of semiquinone anion radical of DHPA calculated by PM3 as a semiemperical hamiltonian method.

Fig. 3. The suggested mechanism for the photochemically initiated luminol CL reaction of quinones.

Fig. 4. Effects of different luminol analogues on (A) CL intensity and (B) S/N ratio. Samples concentrations were $50 \mathrm{nM}$ from each quinone.

Fig. 5. Effects of concentration of L-012 reagent on (A) CL intensity and (B) S/N ratio. Samples concentrations were $50 \mathrm{nM}$ from each quinone.

Fig.6. A typical chromatogram of a standard mixture of quinones (50 nM) obtained using the proposed CL method. Peaks: $1=1,2-\mathrm{NQ} ; 2=1,4-\mathrm{NQ} ; 3=\mathrm{PQ} ; 4=\mathrm{AQ}$.

Fig. 7. Chromatograms for quinones in the extract from (A) airborne particulates and (B) airborne particulates spiked with $50 \mathrm{nM}$ of a mixture of standard quinones. Peaks: $1=$ 1,2-NQ; 2 = 1,4-NQ; 3 = PQ; 4 = AQ. 
Fig. 1

(A)

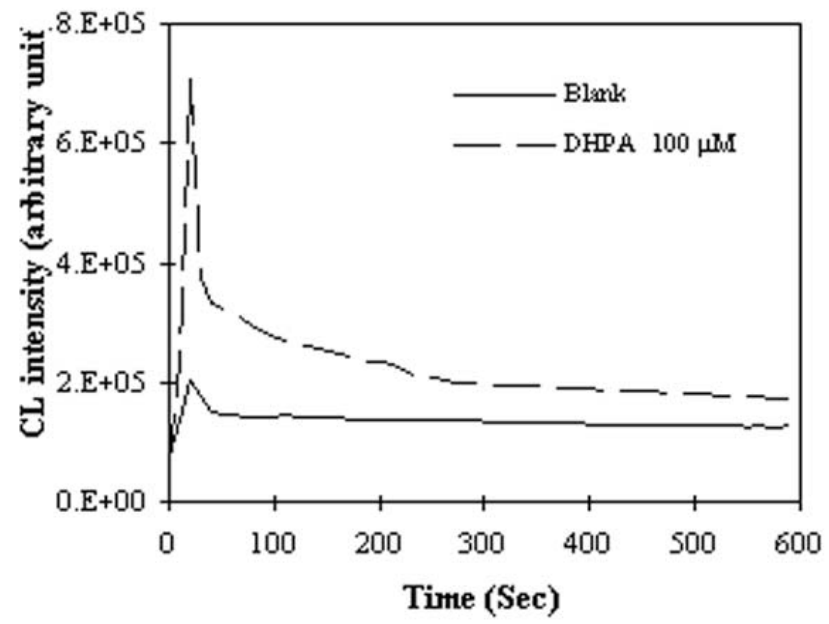

(B)

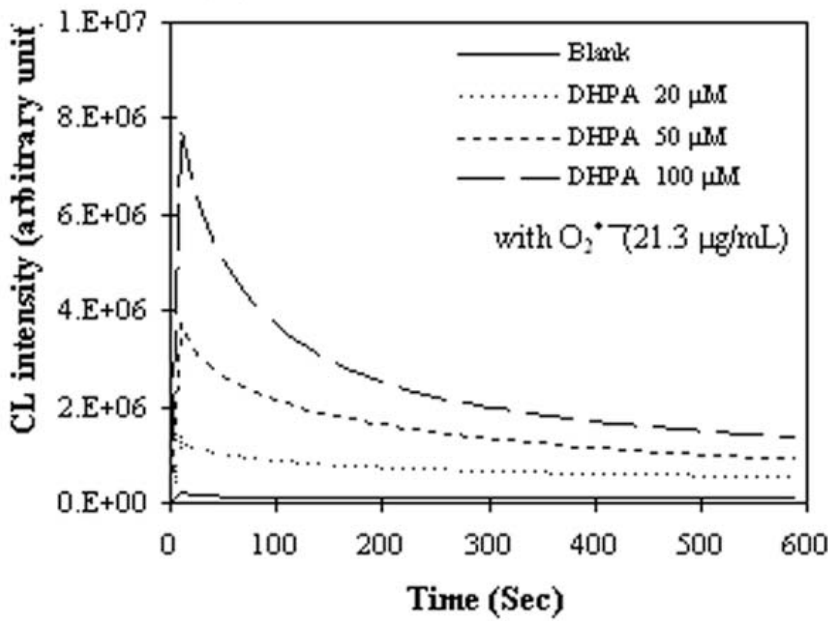

Fig. 2

(A)

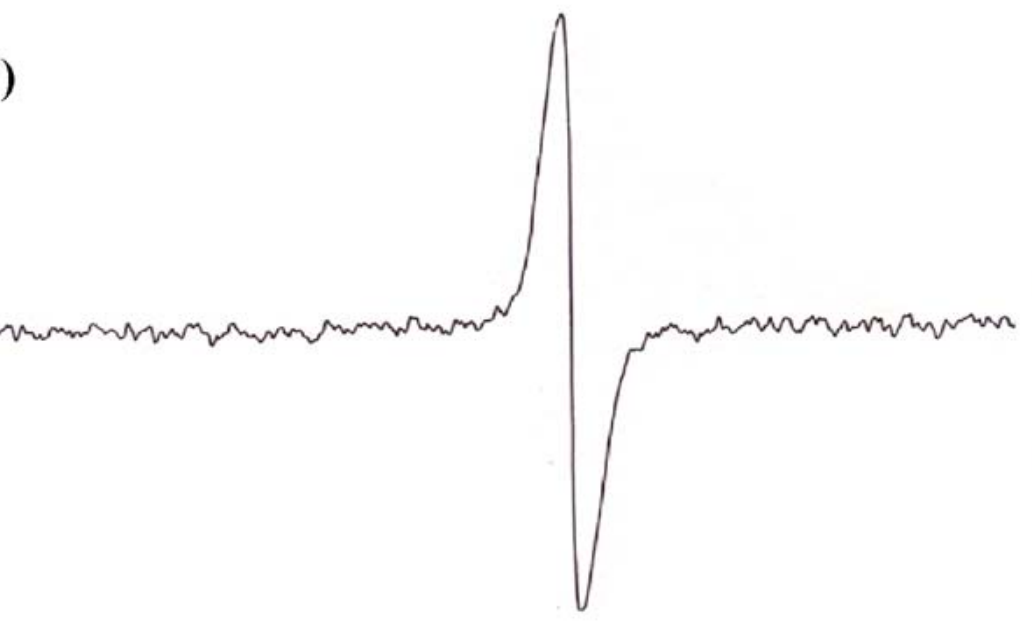

(B)

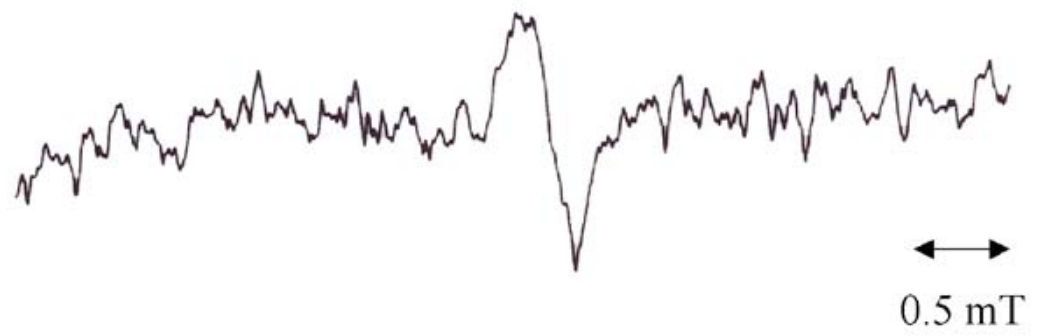

(C)

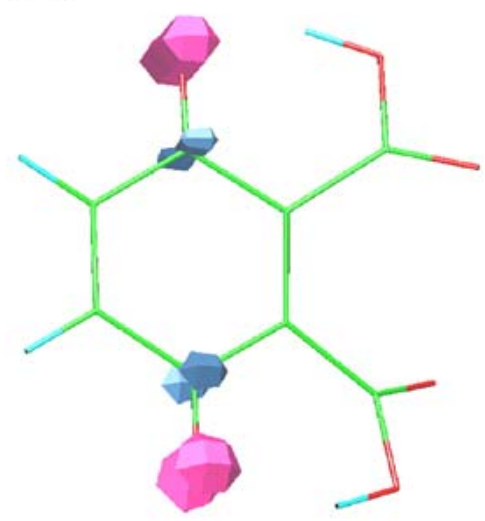


Fig. 3

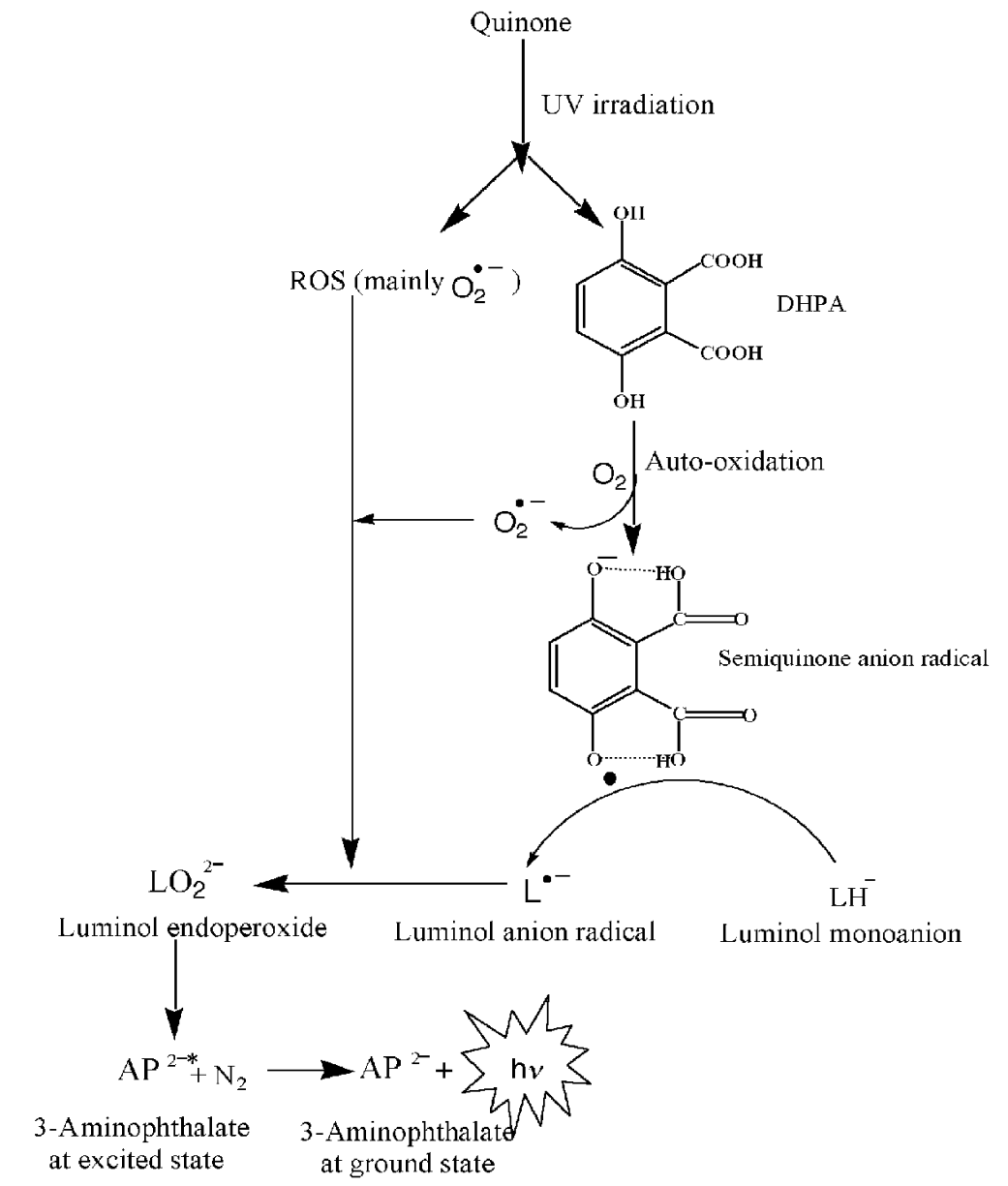

Fig. 4
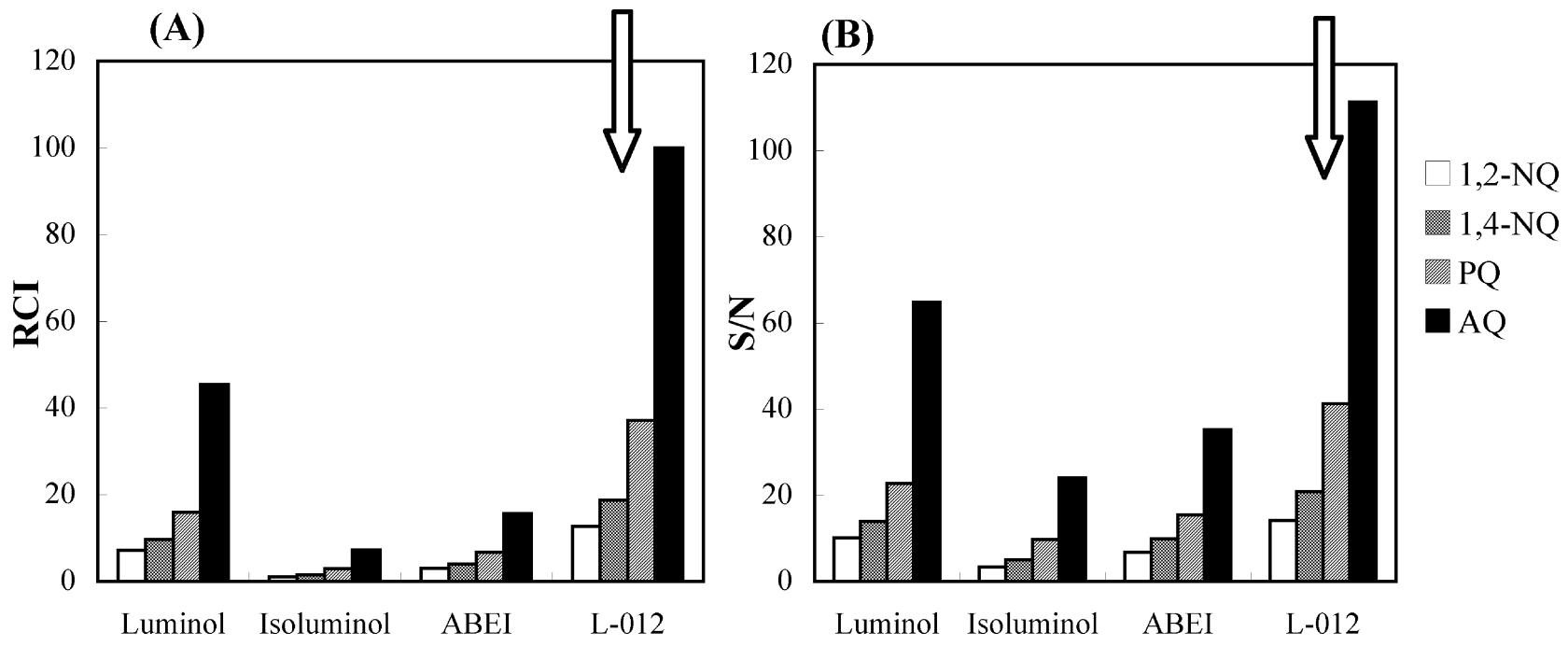
Fig. 5

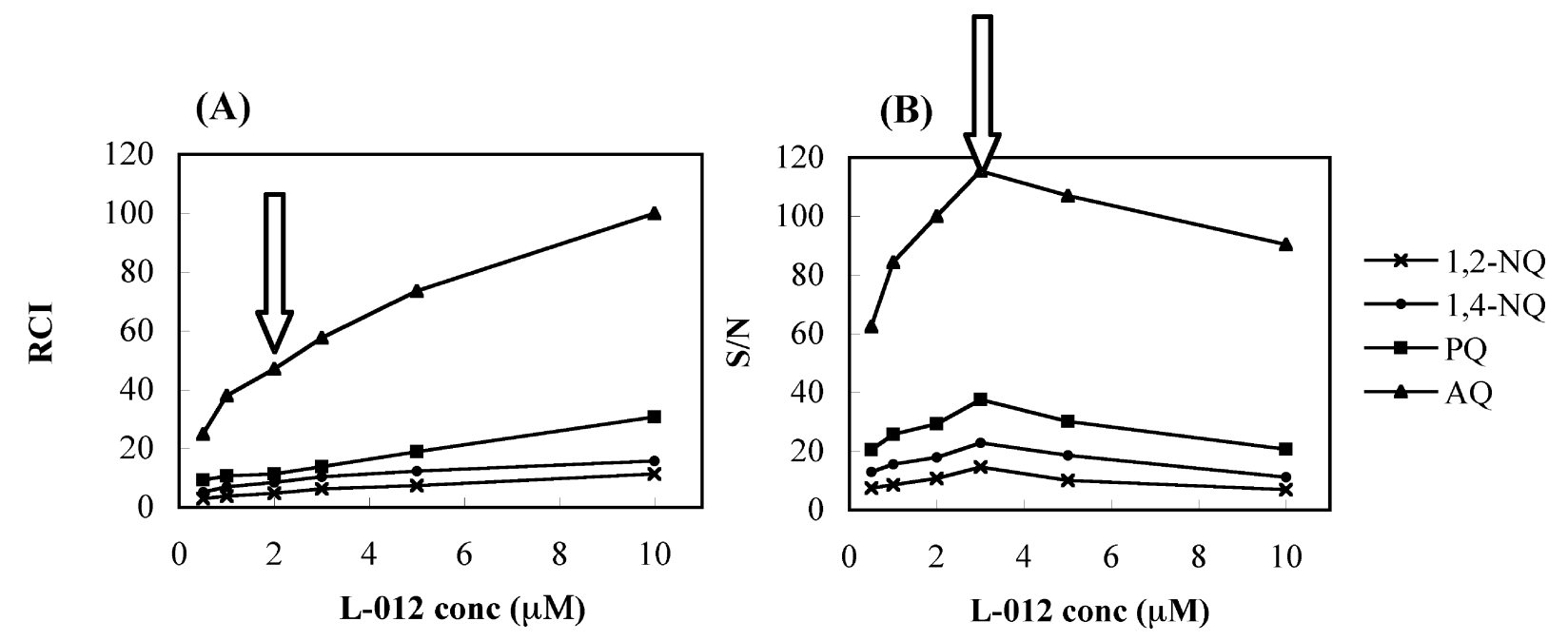

Fig. 6

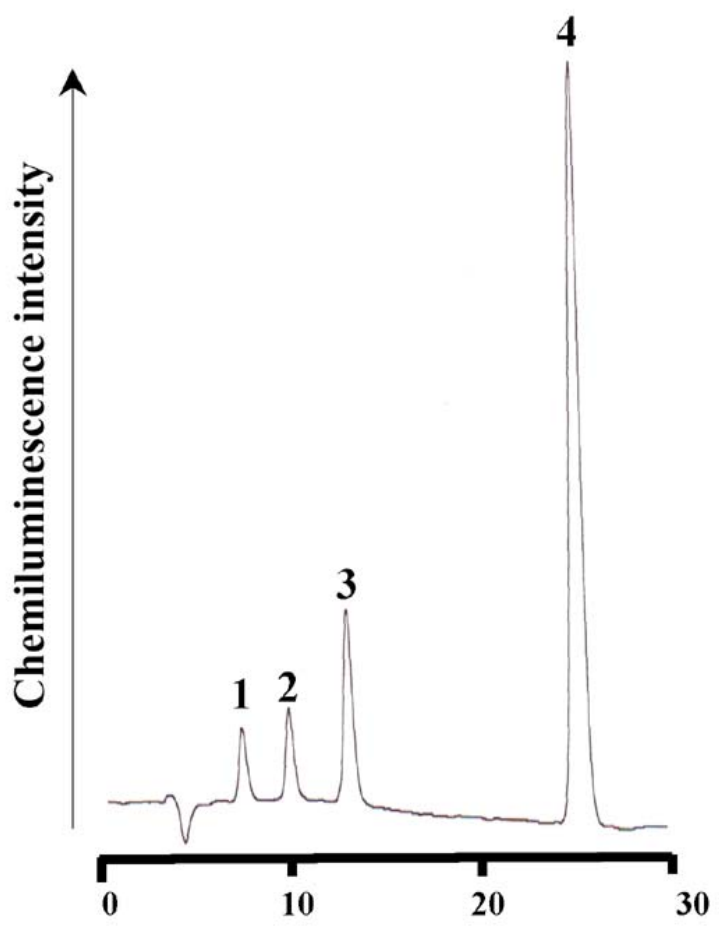

Retention time, min 
Fig. 7

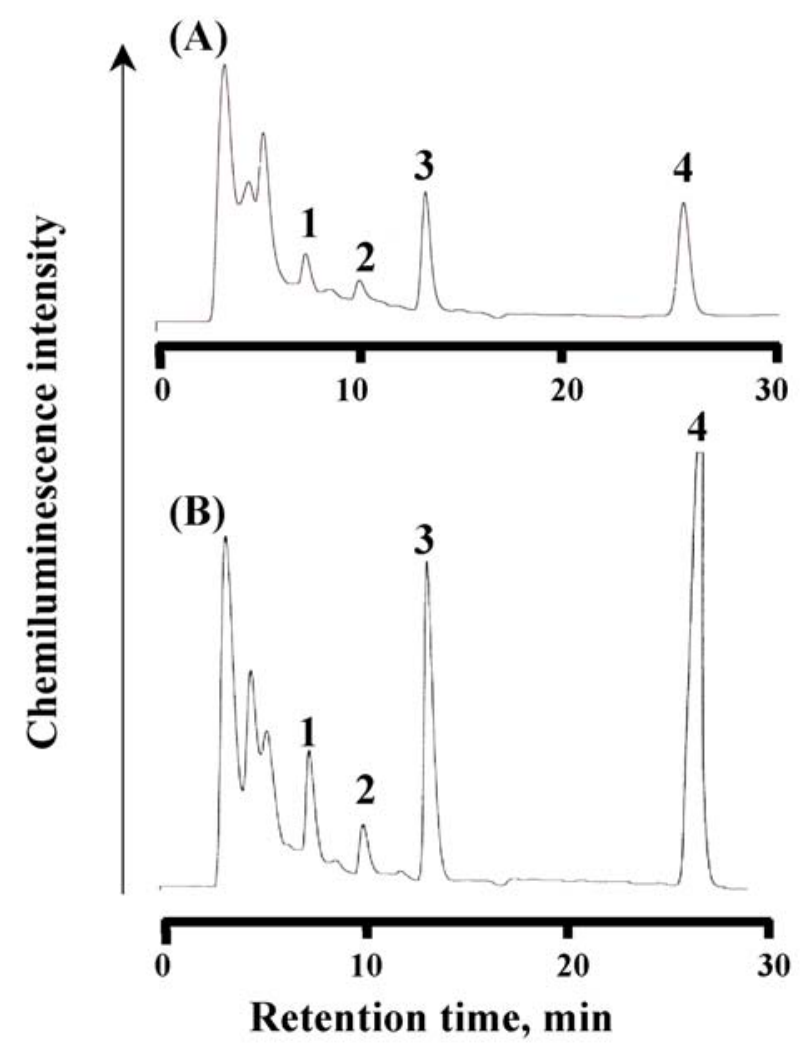


Table 1

Effect of selective ROS scavengers on CL produced by anthraquinone photoproducts and L-012

\begin{tabular}{cccc}
\hline Scavenger & ROS & Concentration & RCI* $^{*}$ \\
\hline Without Scavenger & ----- & ----- & 100 \\
SOD & $\mathrm{O}_{2}^{\bullet-}$ & $1 \mathrm{U} / \mathrm{mL}$ & 18.2 \\
& & $10 \mathrm{U} / \mathrm{mL}$ & 5.6 \\
Mannitol & $10 \mu \mathrm{M}$ & 88.0 \\
& & $100 \mu \mathrm{M}$ & 81.6 \\
$\mathrm{NaN}_{3}$ & $10 \mu \mathrm{M}$ & 84.2 \\
& $100 \mu \mathrm{M}$ & 78.5 \\
\hline
\end{tabular}

* CL intensity of anthraquinone photoproducts with L-012 without scavenger was taken as 100 .

Table 2

Retention times, calibration curves and detection limits for quinones

\begin{tabular}{|c|c|c|c|c|c|c|}
\hline \multirow[b]{2}{*}{ Sample } & \multirow{2}{*}{$\begin{array}{c}t_{\mathrm{R}} \\
(\min )\end{array}$} & \multicolumn{4}{|c|}{ Calibration curve $^{\mathrm{a}}$} & \multirow{2}{*}{$\begin{array}{c}\text { Detection limit }^{\mathrm{b}} \\
\text { f1ol/injection } \\
\text { (nM) }\end{array}$} \\
\hline & & $\begin{array}{l}\text { Range } \\
(\mathrm{nM})\end{array}$ & $\begin{array}{l}\text { Slope }^{c} \\
( \pm \text { SE })\end{array}$ & $\begin{array}{c}\text { Intercept }^{\mathrm{C}} \\
( \pm \mathrm{SE})\end{array}$ & r & \\
\hline 1,2-NQ & 7.4 & $3-1000$ & $0.09( \pm 0.02)$ & $1.8( \pm 0.27)$ & 0.999 & $24(1.20)$ \\
\hline 1,4-NQ & 10.1 & $2-1000$ & $0.10( \pm 0.01)$ & $2.3( \pm 0.31)$ & 0.998 & $16(0.80)$ \\
\hline PQ & 13.5 & $1-1000$ & $0.11( \pm 0.02)$ & $0.4( \pm 0.11)$ & 0.999 & $7(0.35)$ \\
\hline AQ & 26.8 & $0.2-750$ & $0.13( \pm 0.03)$ & $1.3( \pm 0.19)$ & 0.999 & $1.5(0.08)$ \\
\hline
\end{tabular}

${ }^{a}$ Relative chemiluminescence intensity (RCI) versus concentration (nM).

${ }^{\mathrm{b}}$ Detection limit at a $\mathrm{S} / \mathrm{N}$ ratio of 3

${ }^{\mathrm{c}}$ Data presented as mean $\pm \mathrm{SE}$ of three experiments 